\title{
Mutations in the TSC1 gene account for a minority of patients with tuberous sclerosis
}

\author{
Johari B M Ali, Tiina Sepp, Susannah Ward, Andrew J Green, John R W Yates
}

\begin{abstract}
Tuberous sclerosis (TSC) is an autosomal dominant disorder characterised by tumour-like malformations (hamartomas) of the brain, skin, and other organs, often associated with seizures and learning disability. There is genetic heterogeneity with loci for TSC on chromosomes 9q34 (TSC1) and 16p13.3 (TSC2). The recently cloned TSC1 gene has 23 exons spanning some $40 \mathrm{~kb}$ of genomic DNA with an $8.6 \mathrm{~kb}$ transcript. We now report the results of mutation screening by SSCP and heteroduplex analysis of genomic DNA for all 21 coding exons of TSC1 in 83 unrelated cases of tuberous sclerosis.

TSC1 gene mutations were found in 16 of the 83 cases (19\%). These comprised base substitutions, small insertions, or small deletions giving rise to six nonsense mutations, eight frameshifts, and two splice site mutations, all of which would be expected to result in a truncated or absent protein. In the 10 cases predicted to have TSC1 mutations by linkage analysis or loss of heterozygosity studies, the mutation was identified in eight $(80 \%)$. In the remaining 73 unassigned cases, only eight mutations were found (11\%). From these data we estimate that TSC1 mutations accounted for $24 \%$ of the cases in this sample (and an estimated $22 \%$ of all TSC cases). This contrasts with data from linkage studies suggesting that TSC1 and TSC2 mutations account for approximately equal numbers of families. $(\mathcal{H}$ Med Genet 1998;35:969-972)
\end{abstract}

Keywords: tuberous sclerosis; TSC1 gene; mutation detection

Tuberous sclerosis (TSC) is an autosomal dominant condition characterised by tumourlike malformations (hamartomas) of the skin, brain, heart, kidney, and other organs. Most patients have seizures and a minority have learning disability. ${ }^{1}$ The birth incidence is 1 in 10000 or higher. ${ }^{2}$ In two-thirds of cases the parents are unaffected (so called sporadic cases) and the disease is the result of a new dominant mutation. Linkage studies have identified loci for TSC on chromosomes 9q34
(TSC1) and $16 \mathrm{p} 13.3$ (TSC2) and suggest that they account for approximately equal numbers of families. ${ }^{3}$

The TSC2 gene was characterised in $1993 .^{4}$ Both truncating and missense mutations are common and large deletions of 1-5 kb have also been reported..$^{40}$ The gene has 41 exons spanning approximately $45 \mathrm{~kb}$ of genomic DNA. The $5.5 \mathrm{~kb}$ mRNA encodes the protein tuberin, which has a region of sequence homology to the GTPase activating protein rap1GAP. ${ }^{4}$ The finding of loss of heterozygosity in TSC hamartomas suggests that tuberin acts as a tumour suppressor. ${ }^{11}$

The TSC1 gene was first mapped to chromosome $9 \mathrm{q} 34$ in $1987 .{ }^{12}$ Loss of heterozygosity (LOH) in the TSC1 region in hamartomas suggests that the gene product also acts as a tumour suppressor. ${ }^{13} 14$ However, LOH for the TSC1 region is significantly less common than for TSC $2 . .^{15}$ In a concerted effort by a Consortium of six research groups, the TSC1 gene has recently been isolated by a combination of positional cloning and large scale sequencing. ${ }^{17}$ The gene spans $40 \mathrm{~kb}$ with 23 exons and an $8.6 \mathrm{~kb}$ mRNA. There are $21 \mathrm{cod}-$ ing exons, the largest being exons 15 (559 bp) and 23 ( $517 \mathrm{bp}$ of coding sequence) and the remainder varying in size from 44 to $188 \mathrm{bp}$. The TSC1 protein, hamartin, contains 1164 amino acids with a predicted mass of $130 \mathrm{kDa}$.

In the Consortium publication, eight exons were analysed in a large panel of TSC cases. We now present the results of screening all 21 coding exons of the TSC1 gene for mutations in 83 unrelated UK patients.

\section{Methods}

PATIENTS

Patients were selected from a panel of 93 cases of tuberous sclerosis fulfilling the diagnostic criteria proposed by Gomez. ${ }^{18}$ Four familial cases and six sporadic cases were excluded because they had previously been shown to have TSC2 mutations ${ }^{9}$ or there was linkage or other evidence to assign them to TSC2. The remaining 83 patients comprised 23 familial and 60 sporadic cases. Ten were predicted to have TSC1 mutations on the basis of either (1) exclusion of TSC2 by linkage analysis with affected relatives having different $16 \mathrm{p} 13.3$ haplotypes, or (2) evidence of linkage to $9 q 34$

\author{
Received 5 March 1998 \\ Revised version accepted for \\ publication 22 May 1998 \\ Department of Paediatrics,
University College \\ National College Dublin, \\ Genetics, Our Lady's \\ Hospital, Crumlin, Dublin,
}


Table 1 Pathogenic mutations identified in the TSC1 gene

\begin{tabular}{lllll}
\hline Mutn No & Mutation & Location & Mutation type & Family category \\
\hline 1 & $585-1$ G>C & Intron 5 & Splice acceptor & S \\
2 & 875 insA & Exon 7 & Frameshift & F \\
3 & 1122 C $>$ T; Q301X & Exon 9 & Nonsense & F (TSC1) \\
4 & $1250+1$ G>A & Intron 10 & Splice donor & F (TSC1) \\
5 & 1424delT & Exon 12 & Frameshift & F (TSC1) \\
6 & 1552 C>G; S444X & Exon 13 & Nonsense & F (TSC1) \\
7 & 1746 C>T; R509X & Exon 15 & Nonsense & S \\
8 & 1938 C>T; Q573X & Exon 15 & Nonsense & S \\
9 & 1995insGA & Exon 15 & Frameshift & S (TSC1) \\
10 & 2105 delAAAG & Exon 15 & Frameshift & S \\
11 & 2332 delAT & Exon 17 & Frameshift & S (TSC1) \\
12 & 2395 insA & Exon 17 & Frameshift & F (TSC1) \\
13 & 2519 del23bp & Exon 18 & Frameshift & S \\
14 & 2577 C>T; R786X & Exon 18 & Nonsense & F \\
15 & 2718 C>T; Q833X & Exon 19 & Nonsense & F (TSC1) \\
16 & 2887 insA & Exon 21 & Frameshift & S \\
\hline
\end{tabular}

${ }^{\star} \mathrm{F}=$ familial case; $\mathrm{S}=$ sporadic case $\mathrm{TSC} 1$ = predicted to have $\mathrm{TSC} 1$ mutation from linkage data or loss of heterozygosity studies. Mutation Nos 7, 9-14 have been reported previously. ${ }^{17}$

markers with a lod score of at least 3 , or (3) loss of heterozygosity for $9 q 34$ markers in a TSC hamartoma. In familial cases, samples from affected relatives were used to test whether mutations segregated with the disease. In sporadic cases, the normal parents were tested for mutations identified in their offspring.

PCR AMPLIFICATION

DNA was extracted from peripheral blood lymphocytes using standard methods. ${ }^{19}$ Exons 3-23 of the TSC1 gene were individually amplified from genomic DNA by PCR to give products in the size range 150 to $250 \mathrm{bp}$ suitable for SSCP. Exon 15 was amplified in two overlapping segments of approximately $330 \mathrm{bp}$. Exon 23 was amplified in four overlapping segments of approximately $220 \mathrm{bp}$. Primer sequences are available from the web site http://expmed.bwh.harvard.edu/projects/tsc/.
A

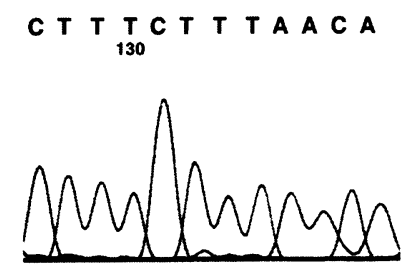

$\mathbf{B}$
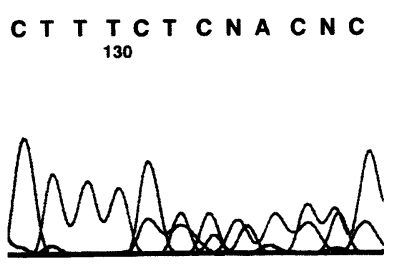

C

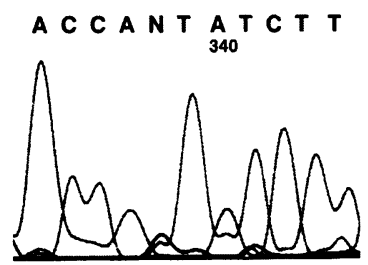

D

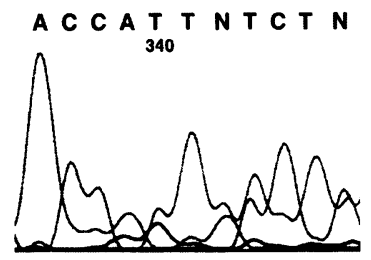
Figure 1 Germline mutation 2105 delAAAG and in the same patient the somatic
mutation 1957delG in a renal cell carcinoma. Automated sequencing of lymphocyte DNA from $(A)$ a normal control showing reverse sequence CTTTCTTTAACA, (B) patient showing CTTT followed by heterozygous sequence owing to deletion of the CTTT repeat in the mutant allele. At the site of the somatic mutation, automated sequencing showed $(C)$ in lymphocyte DNA the normal sequence ACCAGTATCTT, (D) in renal cell carcinoma $D N A$ the sequence ACCA followed by heterozygous sequence owing to deletion of $G$ in the mutant allele. To confirm that the somatic mutation had occurred on the wild type allele, allele specific amplification of the tumour DNA was carried out using a primer specific for the normal sequence at the site of the $2105 \operatorname{del} A A A G$ mutation; automated sequencing of the product confirmed the presence of the $1957 \mathrm{del} G$ mutation.
SSCP AND HETERODUPLEX ANALYSIS

PCR products were screened for mutations by single strand conformational polymorphism $(\mathrm{SSCP})^{20}$ and heteroduplex analysis. ${ }^{21}$ All PCR products showing variant SSCP or heteroduplex patterns were sequenced.

For the SSCP analysis $1 \mu$ l of PCR product was mixed with $9 \mu$ l of loading buffer (95\% formamide, $0.1 \%$ bromophenol blue, $0.1 \%$ xylene cyanol, $10 \mathrm{mmol} / 1 \mathrm{NaOH})$, heat denatured, snap cooled, and loaded onto a $1 \times$ $\mathrm{MDE}^{\mathrm{TM}}$ gel (FMC)/10\% glycerol (v/v) in $1 \times$ TBE. Electrophoresis was carried out at $10^{\circ} \mathrm{C}$ for 16-20 hours at $7 \mathrm{~W}$ using a Protean II xi Cell (BioRad). Bands were visualised by silver staining.

For the heteroduplex analysis, $1 \mu$ of PCR product was mixed with $5 \mu$ l of loading buffer (40\% w/v sucrose, $0.1 \%$ bromophenol blue, $0.1 \%$ xylene cyanol), heat denatured for five minutes, left at $70^{\circ} \mathrm{C}$ for one hour, allowed to cool to room temperature, and loaded onto a 1 $\times \mathrm{MDE}^{\mathrm{TM}}$ gel (FMC) in $1 \times$ TBE. Electrophoresis was carried out at room temperature for 16-20 hours at $400 \mathrm{~V}$. Bands were visualised by silver staining.

\section{AUTOMATED SEQUENCING}

All samples were sequenced using an $\mathrm{ABI}$ Prism $^{\text {TM }} 377$ DNA Sequencer (Perkin Elmer). Dye terminator cycle sequencing kits were provided by Perkin Elmer. For amplification of DNA and subsequent sequencing, the same primers were used as for the original PCR. Purification of templates was done using Wizard $^{\mathrm{TM}}$ Minicolumns (Promega) or QIAquick $^{\mathrm{TM}}$ PCR purification kits (Qiagen). Reaction volumes were halved as compared to ABI protocols. All mutations were confirmed on the opposite strand and from a second PCR product. Mutations were numbered according to the published cDNA sequence. ${ }^{17}$

\section{Results}

Pathogenic TSC1 gene mutations were found in 16 of the 83 patients, representing $19 \%$ of the samples tested $(17 \%$ of the total patient sample including TSC2 mutation cases). Seven of these mutations were included in the Consortium paper. ${ }^{17}$ The mutations are detailed in table 1 . They comprised eight single base substitutions, four small insertions, three small deletions, and one larger deletion of 23 $\mathrm{bp}$, giving rise to six nonsense mutations, eight frameshifts, and two putative splicing mutations. In the patient with the germline mutation 2105delAAAG, analysis of a renal cell carcinoma confirmed the presence of this mutation together with a second mutation, $1957 \mathrm{delG}$, in the other allele which was not present in the germline $^{17}$ (fig 1). All the mutations would be predicted to result in a truncated protein.

Several single base substitutions were found which were not considered to be pathogenic (table 2). The coding polymorphisms 1186 $\mathrm{T}>\mathrm{C}(\mathrm{M} 322 \mathrm{~T})$ in exon 10 and $3050 \mathrm{C}>\mathrm{T}$ (A943A) in exon 22 were observed in several patients and had heterozygosities of $30 \%$ and $18 \%$ respectively in normal controls. The rarer polymorphism $2415 \mathrm{C}>\mathrm{T}$ (H732Y) in exon 17 
Table 2 Polymorphisms identified in the TSC1 gene

\begin{tabular}{llll}
\hline Mutation & Location & $\begin{array}{l}\text { Heterozygosity } \\
\text { (No of controls) }\end{array}$ & Comments \\
\hline $753 \mathrm{G}>\mathrm{A} ; \mathrm{V} 178 \mathrm{I}$ & Exon 7 & $0 \%(100)$ & Not present in affected daughter \\
$1186 \mathrm{~T}>\mathrm{C} ; \mathrm{M} 322 \mathrm{~T}$ & Exon 10 & $30 \%(20)$ & Present in 8\% of patients \\
$2415 \mathrm{C}>\mathrm{T} ; \mathrm{H} 732 \mathrm{Y}$ & Exon 17 & $2 \%(45)$ & Present in unaffected father \\
$2646 \mathrm{G}>\mathrm{C}$; E809Q & Exon 19 & $0 \%(56)$ & Observed in 2 unrelated patients \\
$2867 \mathrm{C}>\mathrm{T} ; \mathrm{A} 882 \mathrm{~A}$ & Exon 21 & $0 \%(67)$ & Present in 20\% of patients \\
$3050 \mathrm{C}>\mathrm{T} ; \mathrm{A} 943 \mathrm{~A}$ & Exon 22 & $18 \%(71)$ & \\
\hline
\end{tabular}

Table 3 Mutation detection rate in sporadic and familial cases predicted to have TSC1 mutations compared to cases where locus unassigned. Number of mutations detected and number of patients screened shown in brackets

\begin{tabular}{llll}
\hline & Sporadic & Familial & Total \\
\hline Predicted TSC1 cases & $67 \%(2 / 3)$ & $86 \%(6 / 7)$ & $80 \%(8 / 10)$ \\
Unassigned cases & $11 \%(6 / 57)$ & $12 \%(2 / 16)$ & $11 \%(8 / 73)$ \\
All cases & $13 \%(8 / 60)$ & $35 \%(8 / 23)$ & $19 \%(16 / 83)$ \\
\hline
\end{tabular}

was present in one patient and in one of 90 control chromosomes. It has previously been reported as a rare variant. ${ }^{22}$ The silent mutation $2867 \mathrm{C}>\mathrm{T}$ (A882A) in exon 21 was observed in two unrelated patients but not in 134 normal chromosomes. This base change would not be expected to have an effect on splicing. Two missense mutations failed to segregate with the disease. One patient had the mutation 753 $\mathrm{G}>\mathrm{A}$ resulting in the substitution of isoleucine for valine at codon 178 . This conservative change was not present in her affected daughter and is likely to be a rare polymorphism of her normal TSC1 allele. Another patient who was a sporadic case had the mutation 2646 $\mathrm{G}>\mathrm{C}$ resulting in the substitution of glutamine for glutamic acid at codon 809 . This mutation was present in his unaffected father.

In the 10 cases predicted to have TSC1 mutations by linkage analysis or loss of heterozygosity studies, eight mutations were identified giving a detection rate of $80 \%$ (table 3 ). In the remaining 73 unassigned cases, eight mutations were found, giving a detection rate of only $11 \%$. From these data we estimate that TSC1 mutations account for $24 \%$ of the patients tested and $22 \%$ of the whole patient panel including cases with TSC2 mutations. The overall detection rate in familial cases was $35 \%$ as compared to $13 \%$ in sporadic cases, but the difference did not reach statistical significance (Fisher's exact $p$ value $=0.06$ ).

\section{Discussion}

We have reported the results of mutation screening of the TSC1 gene in 83 unrelated cases of tuberous sclerosis using SSCP and heteroduplex analysis to test all 21 coding exons amplified from genomic DNA. Mutations were detected in $19 \%$ of the patients tested (representing $17 \%$ of the whole patient panel including cases with TSC2 mutations). This is comparable to the study by Jones et $a l^{2}$ who identified mutations in $13 \%$ of their patients using a similar approach. The mutations were either single base substitutions, small deletions, or insertions giving rise to nonsense mutations, frameshifts, or aberrant splicing. This is similar to the mutation spectrum reported by others. ${ }^{17} 22$

Three-quarters of the mutations identified were novel changes only observed in a single family and these data bring to 54 the total number of different mutations reported. ${ }^{172}$ This illustrates the great diversity of mutations that occur in the TSC1 gene. The recurrent mutations were 2105 delAAAG reported in six unrelated families, $2577 \mathrm{C}>\mathrm{T}$ in four families, $1746 \mathrm{C}>\mathrm{T}$ in three families, and 2887 ins $\mathrm{A}$ in two unrelated patients. Mutations were distributed throughout the gene with a tendency to cluster in exon 15, the largest exon. Mutations have now been reported in the majority of the coding exons, the exceptions being exons 3,8 , $14,16,22$, and $23 .{ }^{172}$ During the course of this study several coding polymorphisms were also identified (table 2). Of these, only two occurred at an appreciable frequency, $1186 \mathrm{~T}>\mathrm{C}$ (M322T) in exon 10 with a heterozygosity of $30 \%$ and $3050 \mathrm{C}>\mathrm{T}$ (A943A) in exon 22 with a heterozygosity of $18 \%$. Both have been reported previously. ${ }^{22}$

All the mutations described here, in common with the majority of reported mutations, would be predicted to result in a truncated protein with probable loss of protein function. This is consistent with the hypothesis that TSC1 acts as a tumour suppressor gene. Additional support for this comes from the analysis of tumours from two patients in whom we identified the germline mutation and had previously shown loss of heterozygosity for TSC1 markers in the lesions. ${ }^{13}{ }^{15}$ In one case with the germline mutation 1995insGA, we were able to confirm that in the hamartoma (a giant cell astrocytoma) only the mutant allele was present. In another patient with the germline mutation 2105delAAAG, analysis of a renal cell carcinoma confirmed the presence of this mutation together with a separate inactivating somatic mutation $1957 \mathrm{delG}$ of the wild type allele ${ }^{17}$ (fig 1). This directly implicates the TSC1 gene in the pathogenesis of the tumour.

Only two missense mutations have been reported in the TSC1 gene. This is in contrast to TSC2 where missense mutations are relatively common. ${ }^{4-10}$ There are other important differences in the mutation spectrum. No large scale germline rearrangements have been observed in TSC1, whereas large deletions of TSC2 occur in up to $5 \%$ of cases. ${ }^{4}$ Similarly, in TSC hamartomas, large somatic deletions encompassing TSC1 are rare, whereas somatic TSC2 gene deletions occur in about $30 \%$ of lesions. ${ }^{1516}$

The identification of the TSC1 gene is an important step in providing a molecular genetic diagnostic service for families affected by TSC. However, screening of 21 exons amplified from genomic DNA is labour intensive and analysis of TSC1 mRNA may be a more efficient approach. Since most TSC1 mutations are truncating, the analysis of TSC1 mRNA by PTT might provide an effective mutation screening strategy. We have previously shown that mutations can be detected in the TSC2 gene by analysis of mRNA using the protein truncation test (PTT). ${ }^{9}$

In developing protocols for mutation screening, important considerations are mutation detection rates and the frequencies of TSC1 and TSC2 mutations in familial and sporadic 
cases. In the current study, there were 10 cases predicted to have TSC1 mutations by linkage analysis or loss of heterozygosity studies and eight mutations were identified, giving an estimated mutation detection rate for TSC1 mutants of $80 \%$. This would be comparable to the detection rates achieved with SSCP/ heteroduplex analysis in studies of other genes. On the assumption that the same detection rate applies to the unassigned cases where another eight mutations were found, we would estimate that two TSC1 mutations were missed. This would give an estimated 20 TSC 1 mutants in the sample of 83 patients, representing $24 \%$ of the patients tested and $22 \%$ of the whole patient panel including cases with TSC2 mutations. This is at odds with the data from linkage studies suggesting that TSC1 and TSC2 contribute equally to familial cases of tuberous sclerosis. ${ }^{3}$ In the Consortium paper, the mutation detection rate in patients predicted to have TSC1 mutations by linkage analysis was also substantially higher than in unassigned cases. ${ }^{17}$ Jones et $a l^{22}$ reported a significant under-representation of TSC1 mutations in sporadic cases, finding mutations in $37 \%(9 / 24)$ of familial cases as compared to $9 \%(13 / 147)$ of sporadic cases. To account for this, they suggested that the mutation rate in the TSC2 gene is higher than in TSC1, but mutations in TSC2 are associated with a lower reproductive fitness, giving rise to more equal representation in families. In the present study, the comparable figures for TSC1 mutation detection were $35 \%(8 / 23)$ in familial cases and $13 \%(8 / 60)$ in sporadic cases (table 3$)$, showing the same trend but failing to reach statistical significance. More data will be needed to determine whether the under-representation of TSC1 mutations is confined to sporadic cases or also applies to families.

We thank the many families who provided samples for th research and the clinicians who helped with ascertainment. We are grateful for financial support from The University of Malaya (JBMA), the Tuberous Sclerosis Association of Great Britain (TS), and the Westminster Medical School Research Trust (Royds Fund) (SW).

1 Gomez MR, ed. Tuberous sclerosis. 2nd ed. New York: Raven Press, 1988.
2 Osborne JP, Fryer A, Webb D. Epidemiology of tuberous sclerosis. Ann NY Acad Sci 1991;615:125-7.

3 Povey S, Burley MW, Attwood J, et al. Two loci for tuberous sclerosis: one on 9q34 and one on 16p13. Ann Hum Genet 1994;58:107-27.

4 European Chromosome 16 Tuberous Sclerosis Consortium. Identification and characterization of the tuberous tium. Identification and characterization of the tuberous

5 Kumar A, Wolpert C, Kandt RS, et al. A de novo frame-shift mutation in the tuberin gene. Hum Mol Genet 1995;4:1471

6 Kumar A, Kandt RS, Wolpert C, Roses AD, Pericak-Vance MA, Gilbert JR. Mutation analysis of the TSC2 gene in an African-American family. Hum Mol Genet 1995;4:2295-8.

7 Vrtel R, Verhoef S, Bouman K, et al. Identification of a nonsense mutation at the 5 ' end of the TSC2 gene in a family with a presumptive diagnosis of tuberous sclerosis com with a presumptive diagnosis of
plex. F Med Genet 1996;33:47-51.

8 Wilson PJ, Ramesh V, Kristiansen A, et al. Novel mutation detected in the TSC2 gene from both sporadic and familia TSC patients. Hum Mol Genet 1996;5:249-56.

9 van Bakel I, Sepp T, Ward S, Yates JRW, Green AJ. Mutations in the TSC2 gene: analysis of the complete coding sequence using the protein truncation test (PTT). Hum Mol Genet 1997;6:1409-14.

10 Au KS, Rodriguez JA, Rodriguez E, Dobyns WB, Delgado $M R$, Northrup $H$. Mutations and polymorphisms in the tuberous sclerosis complex gene on chromosome 16. Hum Mutat 1997;9:23-9.

11 Green AJ, Smith M, Yates JRW. Loss of heterozygosity on chromosome $16 \mathrm{p} 13.3$ in hamartomas from tuberous sclerosis patients. Nat Genet 1994;6:192-6.

12 Fryer AE, Chalmers A, Connor JM, et al. Evidence that the gene for tuberous sclerosis is on chromosome 9. Lancet 1987;i:659-61.

13 Green AJ, Johnson PH, Yates JRW. The tuberous sclerosis gene on chromosome $9 \mathrm{q} 34$ acts as a growth suppressor. Hum Mol Genet 1994;3:1833-4.

14 Carbonara C, Longa L, Grosso E, et al. $9 \mathrm{q} 34$ loss of heterozygosity in a tuberous sclerosis astrocytoma suggests growth suppressor-like activity also for the TSC1 gene. Hum Mol Genet 1994;3:1829-32.

15 Sepp T, Yates JRW, Green AJ. Loss of heterozygosity in tuberous sclerosis hamartomas. $\mathcal{f}$ Med Genet 1996;33:962

16 Henske LP, Scheithauer BW, Short MP, et al. Allelic loss is frequent in tuberous sclerosis kidney lesions but rare in brain lesions. Am $\mathcal{F}$ Hum Genet 1996;59:400-6.

17 The TSC1 Consortium. Identification of the tuberous sclerosis gene TSC1 on chromosome 9q34. Science 1997;277: 805-8.

18 Gomez MR. Phenotypes of the tuberous sclerosis complex with a revision of diagnostic criteria. Ann NY Acad Sci 1991;615:1-7.

19 Maniatis TM, Fritsch EF, Sambrook J. Molecular cloning: laboratory manual. New York: Cold Spring Harbour Press, 1982

20 Orita M, Suzuki Y, Sekiya T, Hayashi K. Rapid and sensitive detection of point mutations and DNA polymorphisms using the polymerase chain reaction. Genomics 1989;5:8749.

21 Ganguly A, Rock MJ, Prockop DJ. Conformation-sensitive gel electrophoresis for rapid detection of single-base differences in double-stranded PCR products and DNA fres in : evidence for solvent-induced bends in DNA heteroduplexes. Proc Natl Acad Sci USA 1993;90:10325-9.

22 Jones AC, Daniells CE, Snell RG, et al. Molecular genetic and phenotypic analysis reveals differences between TSC and TSC 2 associated familial and sporadic tuberous sclerosis. Hum Mol Genet 1997;6:2155-61. 